\title{
Principal Component Analysis of Diffusion Tensor Images to Determine White Matter Injury Patterns Underlying Postconcussive Headache
}

\author{
(D) A. Ghodadra, (D) L. Alhilali, and (D) S. Fakhran
}

\begin{abstract}
BACKGROUND AND PURPOSE: Principal component analysis, a data-reduction algorithm, generates a set of principal components that are independent, linear combinations of the original dataset. Our study sought to use principal component analysis of fractional anisotropy maps to identify white matter injury patterns that correlate with posttraumatic headache after mild traumatic brain injury.
\end{abstract}

MATERIALS AND METHODS: Diffusion tensor imaging and neurocognitive testing with the Immediate Post-Concussion Assessment and Cognitive Test were performed in 40 patients with mild traumatic brain injury and 24 without posttraumatic headache. Principal component analysis of coregistered fractional anisotropy maps was performed. Regression analysis of the major principal components was used to identify those correlated with posttraumatic headache. Finally, each principal component that correlated with posttraumatic headache was screened against other postconcussive symptoms and demographic factors.

RESULTS: Principal component 4 (mean, $7.1 \pm 10.3$ ) correlated with the presence of posttraumatic headache in mild traumatic brain injury (odds ratio per SD, 2.32; 95\% CI, 1.29-4.67; $P=.01$ ). Decreasing principal component 4 corresponded with decreased fractional anisotropy in the midsplenium and increased fractional anisotropy in the genu of the corpus callosum. Principal component 4 identified patients with posttraumatic headache with an area under the receiver operating characteristic curve of 0.73 and uniquely correlated with posttraumatic headache and no other postconcussive symptom or demographic factors.

CONCLUSIONS: Principal component analysis can be an effective data-mining method to identify white matter injury patterns on DTI that correlate with clinically relevant symptoms in mild traumatic brain injury. A pattern of reduced fractional anisotropy in the splenium and increased fractional anisotropy in the genu of the corpus callosum identified by principal component analysis can help identify patients at risk for posttraumatic headache after mild traumatic brain injury.

ABBREVIATIONS: FA = fractional anisotropy; ImPACT = Immediate Post-Concussion Assessment and Cognitive Test; $\mathrm{mTBI}=$ mild traumatic brain injury; $\mathrm{PC}=$ principal component; $\mathrm{PCA}=$ principal component analysis; $\mathrm{PCS}=$ postconcussive symptoms; $\mathrm{PTH}=$ posttraumatic headache

$\mathbf{M}^{\mathrm{i}}$ ild traumatic brain injury (mTBI), commonly referred to as "concussion," is a far-reaching disease, affecting up to 1.7 million individuals in the United States annually. ${ }^{1}$ Many of these patients have chronic neurologic symptoms that profoundly impact daily life, ${ }^{2}$ with approximately $15 \%$ of patients having persistent neurologic symptoms beyond 3 months. ${ }^{3}$ This "miserable minority" with persistent symptoms such as headache, fatigue,

Received June 26, 2015; accepted July 2.

From the Department of Radiology, University of Pittsburgh Medical Center, Pittsburgh, Pennsylvania.

Abstract previously presented at: American Society of Neuroradiology Annual Meeting and the Foundation of the ASNR Symposium, April 25-30, 2015; Chicago, Illinois.

Please address correspondence to Anish Ghodadra, MD, University of Pittsburgh Medical Center, Department of Radiology, 200 Lothrop St, Suite 201 East Wing, Pittsburgh, PA 15213; e-mail: ghodadraag@upmc.edu; @AGhodadraMD

http://dx.doi.org/10.3174/ajnr.A4505 photophobia, nausea, and visual deficits ${ }^{4}$ include many individuals in the prime of life with notable repercussions on the quality of life and productivity. ${ }^{5}$

Among postconcussion symptoms (PCS), posttraumatic headache (PTH) is one of the most frequent, enduring, and debilitating symptoms, with estimates of up to $90 \%$ prevalence following mTBI. ${ }^{6}$ The morbidity associated with PTH is high, with poorer neurocognitive test performance, exacerbation of other PCS, and longer recovery times. ${ }^{7}$ Unfortunately, imaging of mTBI and PTH has proved to be difficult because routine CT and MR imaging findings are often negative in these patients. However, recently, diffusion tensor imaging has emerged as a powerful MR imaging technique to identify disruption of major white matter fiber tracts in the brain after trauma ${ }^{8,9}$ and has detected white matter injuries underlying several postconcussion symptoms. ${ }^{1,10}$ While DTI continues to show promise as an imaging tool in detecting injuries underlying $\mathrm{mTBI}$ and PCS, analysis of the large 
DTI datasets traditionally involves complex voxelwise techniques such as tract-based spatial statistics. ${ }^{11}$ Analytic methods of this type have many advantages; however, at best, these methods simply identify regions of the brain in which DTI metrics, such as fractional anisotropy (FA), are different among groups of subjects. As a result, assessment of the correlation among brain regions is more difficult.

In an effort to overcome the limitations of traditional voxelwise methods, we sought to use principal component analysis (PCA) to analyze FA changes in patients with mTBI. PCA is a data-reduction algorithm that generates a set of new variables or principal components (PCs) that are orthogonal linear combinations of the original dataset to maximally explain the variance of the dataset. ${ }^{12}$ This process allows a large number of redundant variables to be condensed into a relatively few new variables, or PCs, that give the most information about the data. These PCs can then be analyzed to determine which ones correlate with outcomes of interest. The purpose of our study was to use PCA of FA maps to identify white matter injury patterns that correlate with PTH after mTBI.

\section{MATERIALS AND METHODS Study Population}

Our institutional review board approved this study, with a waiver of informed consent. All studies included were performed as standard of care, and results were retrospectively reviewed.

We searched our electronic medical record to retrospectively identify MR imaging studies with DTI performed for mTBI. Radiology reports from January 1, 2006, to March 1, 2013, were searched by using keywords "concussion," "mild traumatic brain injury," and "diffusion tensor imaging." Inclusion criteria were 10-50 years of age, witnessed closed head trauma, no focal neurologic deficit, loss of consciousness of $<1$ minute, posttraumatic amnesia of $<30$ minutes, and English language proficiency. Exclusion criteria were a prior neuropsychiatric illness (2 patients), abnormal CT or conventional brain MR imaging findings (3 patients), history of substance abuse (3 patients), lack of DTI (4 patients), lack of neurocognitive assessment ( 6 patients), total symptom score of zero ( 3 patients), or inability to affine align FA images (2 patients). Demographic data collected from the electronic medical record included age and sex, type of trauma (sports injury versus non-sports injury), and any history of a prior concussion as diagnosed by an athletic trainer, neuropsychologist, or other medical personnel at any facility.

Neuropsychological and neurocognitive testing was performed by a neuropsychologist with $>14$ years of experience in treating patients with mTBI. Computerized neurocognitive testing was performed with the Immediate Post-Concussion Assessment and Cognitive Testing (ImPACT), in which a total symptom score was calculated for each patient using a 7-point Likert survey encompassing 22 PCS. Patients were classified as having headaches on the basis of the International Headache Society guidelines ${ }^{13}$ following the postconcussion clinical examination. Time to recovery was defined as when the ImPACT total symptom score was zero or the patient stated that he or she was asymptomatic.

\section{Imaging}

Conventional MR imaging and DTI were performed with a $1.5 \mathrm{~T}$ unit (Signa; GE Healthcare, Milwaukee, Wisconsin) and a standard head coil. Despite the relatively long time span of this study, all patients and controls included in this study underwent the same imaging sequences on the same system, as follows: sagittal and axial T1-weighted (TR, $600 \mathrm{~ms}$; TE, minimum; section thickness, $5 \mathrm{~mm}$; NEX, 1), axial proton attenuation-weighted (TR, 2000-2500 ms; TE, minimum; section thickness, 5 mm; NEX 1), T2-weighted (TR/TE, 2000-2500/84-102 ms; section thickness, 5 $\mathrm{mm}$; NEX, 1), fluid-attenuated inversion recovery (TR/TE, $9000-$ 10,000/149 ms; TI, $2200 \mathrm{~ms}$ ), and diffusion-weighted (single-shot echo-planar sequence; TR, 10,000 ms; TE, minimum; section thickness, $5 \mathrm{~mm}$; matrix, $128 \times 128)$. T2*-weighted gradient recalled-echo (TR/TE, 4400/21 ms; NEX, 1; $90^{\circ}$ flip angle; section thickness, $3 \mathrm{~mm}$ ) or susceptibility-weighted (TR/TE, 37/23 ms; NEX, $1 ; 15^{\circ}$ flip angle; section thickness, $2.4 \mathrm{~mm}$ ) sequences were performed. The FOV ranged from 200 to $240 \mathrm{~mm}$.

DTI was performed with a single-shot echo-planar sequence (TR/TE, 4000/80 ms; NEX, 2; section thickness, 5 mm; $128 \times 128$ matrix; FOV, $260 \mathrm{~mm})$. Diffusion gradients were set in 25 noncollinear directions by using $2 \mathrm{~b}$-values ( $b=0$ and $1000 \mathrm{~s} / \mathrm{mm}^{2}$ ).

\section{Image Analysis}

Fractional anisotropy maps were generated as a measure of white matter integrity by using the fMRI of the Brain Diffusion Toolbox (http://fsl.fmrib.ox.ac.uk/fsl/fslwiki/FDT) as part of the fMRI of the Brain Software Library (FSL; http://www.fmrib.ox.ac.uk/fsl). The FA maps included both gray and white matter and were registered to the Montreal Neurological Institute atlas by using a 12-parameter affine transformation.

A mean FA map was created from the 64 coregistered FA images. For each subject, a new 3D matrix was calculated by subtracting the subject's FA map from the mean FA image. The resulting volumes were subjected to PCA, in which each voxel represented a variable. To facilitate interpretation of the PCs, a mean FA map was generated. Additional maps showing the effect of increasing and decreasing the value of each principal component by 2 SDs were then generated. Analysis was conducted by using a custom script in Matlab (MathWorks, Natick, Massachusetts).

\section{Statistical Analysis}

To prevent overfitting, we performed a multistep regression analysis. Initially, the first 20 PCs were screened with univariate analyses for correlation with the presence of PTH. Subsequently, forward stepwise nominal regression with the PCs whose univariate $P$ values were $<.25$ was used to identify correlation with the presence of PTH. Finally, each PC that correlated with the presence of PTH was screened against demographic factors (age, sex) and other postconcussive symptoms, including sleep-wake disturbances, anxiety, tinnitus, and cervicalgia by using univariate $t$ testing or linear regression when appropriate. Receiver operating characteristic curves were created for PCs and the prediction of PTH. Areas under the receiver operating characteristic curve for PCs and PTH were obtained and interpreted according to the guidelines put forth by Hosmer and Lemeshow: no discrimina- 
Table 1: Demographics by study group ${ }^{a}$

\begin{tabular}{lccc}
\hline & No PTH & PTH & $P$ Value \\
\hline Age (yr) & $17.08(2.99)$ & $17.58(5.87)$ & .7 \\
ImPACT total symptom & $37.46(24.87)$ & $29.20(24.65)$ & .2 \\
score & & & \\
\% Male & $254 \%$ & $278 \%$ & .09 \\
\% Prior concussion & $29 \%$ & $250 \%$ & .12 \\
Time to recovery (days) & $56(70)$ & $55(57)$ & .98 \\
\hline
\end{tabular}

${ }^{a}$ Data are mean (SD) unless otherwise noted.

Table 2: Descriptive statistics and results of univariate regression analysis of PCs and PTH

\begin{tabular}{lrcc}
\hline PC & Mean (SD) & $\begin{array}{c}\text { Regression } \\
\text { Coefficient }\end{array}$ & P Value \\
\hline 1 & $1.35(13.62)$ & -0.002 & .897 \\
2 & $37.81(11.67)$ & -0.006 & .785 \\
3 & $7.59(11.58)$ & -0.036 & .141 \\
$4^{\mathrm{a}}$ & $7.09(10.32)$ & 0.081 & .010 \\
5 & $-14.15(9.68)$ & 0.010 & .712 \\
6 & $30.59(9.61)$ & -0.014 & .621 \\
7 & $-17.36(9.11)$ & -0.011 & .699 \\
8 & $11.23(9.02)$ & -0.051 & .105 \\
9 & $13.66(8.83)$ & -0.033 & .294 \\
10 & $10.29(8.84)$ & -0.003 & .917 \\
11 & $-4.83(8.82)$ & 0.012 & .685 \\
12 & $3.99(8.55)$ & 0.053 & .112 \\
13 & $11.26(8.30)$ & -0.044 & .196 \\
14 & $-16.63(8.15)$ & -0.026 & .419 \\
15 & $-1.70(8.03)$ & 0.015 & .645 \\
16 & $-5.50(8.02)$ & -0.037 & .274 \\
17 & $-3.74(7.92)$ & 0.016 & .627 \\
18 & $-0.33(7.65)$ & -0.051 & .152 \\
19 & $-5.82(7.61)$ & 0.048 & .180 \\
20 & $-9.34(7.74)$ & -0.013 & .691 \\
\hline
\end{tabular}

a Principal component 4 was the only statistically significant predictor for posttraumatic headache

tion (area under the receiver operating characteristic curve $=$ $0.5)$, acceptable discrimination $(0.7 \leq$ area under the receiver operating characteristic curve $\leq 0.8)$, excellent discrimination $(0.8 \leq$ area under the receiver operating characteristic curve $\leq 0.9$ ), and outstanding discrimination (area under the receiver operating characteristic $\geq 0.9) .{ }^{14}$ Statistical analysis was conducted with the software package JMP 11 (SAS Institute, Cary, North Carolina).

\section{RESULTS}

Sixty-four patients with mTBI were included in our study, of which $40(63 \%)$ had PTH. The mean age was $17.4 \pm 5.0$ years, with $69 \%$ male. There was no statistically significant difference in mean age (17.6 versus 17.1 years, $P=.70$ ) or percentage male (54\% versus $78 \%, P=.09$ ) between patients with and without PTH. These data are summarized in Table 1.The median time to presentation was not significantly different in patients with and without PTH (22 versus 24 days, $P=.79$ ). All patients had normal brain MR imaging findings.

Univariate analysis of the first 20 principal components demonstrated only PC 4 (mean, $7.1 \pm 10.3$ ) correlated with the presence of PTH in patients with mTBI. The odds ratio per SD of PC 4 was 2.32 , $(95 \% \mathrm{CI}, 1.29-4.67 ; P=.01)$. Table 2 summarizes the results of univariate analysis of the correlation between PTH and each principal component.

Receiver operating characteristic analysis for PC 4 demonstrated acceptable performance with an area under the curve of
ROC Curve for Post-traumatic Headache using PC 4



FIG 1. Receiver operating characteristic curve for the presence of posttraumatic headache based on principal component 4. Receiver operating characteristic curve analysis demonstrates an acceptable diagnostic performance of FA values described by principal component 4 for the presence of posttraumatic headache, with an area under the curve of 0.73 .

0.73 (Fig. 1). Figure 2 shows the effect of changing principal component 4 on the average FA map by 2 SDs. Lower values of PC 4 primarily indicated relatively decreased FA in the splenium and increased FA in the genu of the corpus callosum and corresponded to increased risk of postconcussive headache. Additionally, there were more subtle decreases within the corticospinal tract with decreasing PC 4 . This principal component uniquely correlated with the presence of PTH and did not correlate with any other postconcussive symptom or demographic factor.

\section{DISCUSSION}

Our results show that PCA of DTI in patients with mTBI can successfully identify patterns of FA that correlate with PTH. PCA of FA maps revealed a unique principal component that correlated with increased risk of PTH in patients with mTBI. Decreased values of this principal component, PC 4, most prominently corresponded to decreased FA in the midsplenium of the corpus callosum and increased FA in the genu.

Previous studies of mTBI by using DTI have used 3 major analysis techniques: selection of a priori ROIs, voxelwise analysis (whole-brain or tract-based spatial statistics), and histogram analysis. ${ }^{15}$ While these techniques have shown promise in the analysis of DTI data, histogram analysis cannot identify focal regions of injury and voxelwise analysis and ROI analysis essentially treat each voxel/region as a unique variable that is tested against a clinical outcome of interest. These methods fail to capture the relationship among multiple regions of the brain. Furthermore, despite attempts at compensation for multiple comparisons, there is a higher risk of type 1 statistical errors.

Our technique builds on previous work investigating changes in white matter integrity in patients with mTBI with postconcussion syndrome that used voxel-based approaches. ${ }^{1,10,15,16}$ These reports have demonstrated white matter abnormalities in patients with mTBI relative to controls ${ }^{17-19}$; however, these differences have not correlated with symptoms. ${ }^{20}$ Similarly, attempts to correlate structural injuries with postconcussive cognitive perfor- 


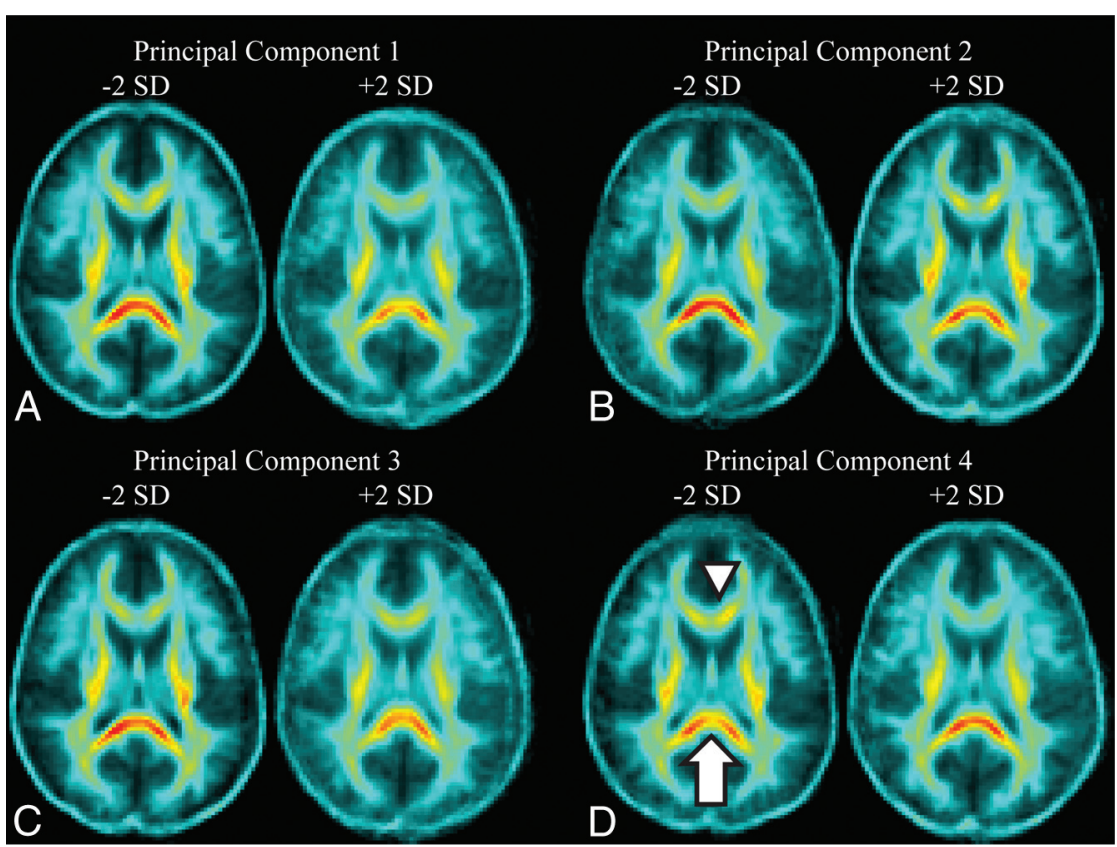

FIG 2. Effect of changing each principal component by \pm 2 SDs on fractional anisotropy in a representative section through the corpus callosum. $A, P C 1$ corresponds to the overall average $F A$ in the brain, with decreasing $P C 1$ resulting in an increase in the overall FA throughout the brain. $B$, PC 2 corresponds most closely with FA in the corticospinal tracts. A decrease in PC 2 results in a noticeable decrease in FA in the corticospinal tracts bilaterally. C, Changes to PC 3 correspond to changes in FA in both the corticospinal tracts and the splenium. Decreases in PC 3 increase the FA in both in the splenium and corticospinal tracts. D, PC 4, the only predictor of posttraumatic headache, corresponds closest to changes in FA in the splenium and genu of the corpus callosum. Decreasing PC 4 results in decreased FA in the splenium of the corpus callosum (white arrow) and increased FA in the genu (white arrowhead).

mance have shown mixed results without a strong anatomic/ pathologic correlation, with deficits detected in regions as diverse as the occipital cortex and corticospinal tracts. ${ }^{21-25}$ This finding suggests that not all regions with differences in FA between patients and controls are necessarily symptomatic. ${ }^{1}$ Reduced FA in 1 region alone may not, in and of itself, be clinically relevant. Given the complex interconnectivity within the brain, it may not be sufficient to simply identify individual, isolated regions of FA variance. Rather, it would be more meaningful to view changes in FA in 1 region in the context of potentially related changes elsewhere in the brain. In short, identifying unique patterns of FA changes in multiple brain regions is needed, rather than merely identifying isolated regions of FA variance.

Our application of PCA to FA maps provides a novel analysis method of complex DTI data as a means of identifying patterns of change in FA. These data-reduction techniques allow distilling complex relationships between variables, in this case FA throughout the brain, to a relatively small set of principal components. Each principal component describes a unique way in which the variables "move" together. Furthermore, to aid in recognition of disease in a clinical setting, these PCs can be visualized in an image (eg, Fig 2).

The finding of a unique principal component that corresponds to FA abnormalities in the genu and splenium of the corpus callosum offers a means of stratifying patients with postconcussive syndrome into groups with high and low risk of the development of posttraumatic headache. Identification of patients at risk for the development of PTH would potentially allow early intervention in an attempt to improve symptoms and outcome, because early comprehensive treatment of PTH has been shown to significantly reduce both the frequency of PTH and headache-related disability.

Additionally, the areas of injury underlying PTH identified by PCA may help elucidate the pathophysiology underlying headache after mTBI. Identifying injury in the splenium is not surprising after mTBI because it is at high risk of direct impact by the falx cerebri and tentorium during trauma. ${ }^{26}$ However, most interesting, abnormalities of this region are also seen in other forms of chronic headache, such as migraine. ${ }^{27,28}$ In fact, in nontraumatic migraine, abnormalities of the splenium are associated with a more chronic disease course, greater headache frequency, and comorbid neuropsychiatric conditions. ${ }^{15}$

Lower values of PC 4 also corresponded to increased FA in the genu of the corpus callosum. This could represent compensatory increases in FA in the genu related to the decreased FA/injury identified in the splenium. Compensatory increases in FA in areas of the corpus callosum in response to other callosal injuries have been seen in many pathologies, including schizophrenia, white matter injury from prematurity, and agerelated white matter loss. ${ }^{29-31}$ The fact that PC 4 represents not only decreased FA in the splenium but also increased FA in the genu may indicate that injury to the splenium only results in PTH when it is substantial enough to trigger compensatory increases in FA in the genu. This pattern of FA identified by PC4 highlights the importance of detecting not simply differences in FA but relationships of changes in FA in different brain regions. PC 4 is not only a marker of FA in the splenium and genu, but rather it represents a unique pattern of FA in the entire brain and describes a complex relationship among these values. Further analysis of FA in the genu and splenium may yield more refined markers for identification of patients at risk for developing PTH.

While our study shows the potential of PCA in FA analysis, there are a few limitations of our findings. First, our study population consisted of a single-center retrospective cohort; thus, larger, multicenter prospective studies are needed to confirm these findings. Second, while our cohort is relatively large, given the relatively large number of voxels and the complexity of the FA structure, larger sample sizes would allow generation of more refined principal components. Furthermore, the registration process, while robust, introduces its own noise into the FA maps that can bias the principal components, particularly along edges. Finally, our analysis focused on a single time point after initial injury. The relationship we found between FA in the corpus callosum and PTH could be confounded by a more longitudinal process of injury. Applying this PCA tech- 
nique to a longitudinal dataset would provide a more robust analysis and could shed light on the pathophysiology of postconcussive headache.

\section{CONCLUSIONS}

PCA can be used as a data-mining method to identify white matter injury patterns on DTI that correlate with clinically relevant symptoms in mTBI. PCA of FA maps in patients with mTBI identified a pattern of reduced FA in the splenium and increased FA in the genu of the corpus callosum that correlates with postconcussive headache in patients with mTBI. Our results suggest that analysis of the FA patterns in the corpus callosum may offer a means of identifying patients at risk for the development of PTH and thus allow early treatment.

\section{REFERENCES}

1. Fakhran S, Yaeger K, Alhilali L. Symptomatic white matter changes in mild traumatic brain injury resemble pathologic features of early Alzheimer dementia. Radiology 2013;269:249-57 CrossRef Medline

2. Bohnen N, Jolles J, Twijnstra A. Neuropsychological deficits in patients with persistent symptoms six months after mild head injury. Neurosurgery 1992;30:692-95; discussion 695-96 CrossRef Medline

3. Shenton ME, Hamoda HM, Schneiderman JS, et al. A review of magnetic resonance imaging and diffusion tensor imaging findings in mild traumatic brain injury. Brain Imaging Behav 2012;6:137-92 CrossRef Medline

4. Ganti L, Khalid H, Patel PS, et al. Who gets post-concussion syndrome? An emergency department-based prospective analysis. Int J Emerg Med 2014;7:31 CrossRef Medline

5. Emanuelson I, Andersson Holmkvist E, Björklund R, et al. Quality of life and post-concussion symptoms in adults after mild traumatic brain injury: a population-based study in western Sweden. Acta Neurol Scand 2003;108:332-38 CrossRef Medline

6. Kontos AP, Elbin RJ, Lau B, et al. Posttraumatic migraine as a predictor of recovery and cognitive impairment after sport-related concussion. Am J Sports Med 2013;41:1497-504 CrossRef Medline

7. Erickson JC. Treatment outcomes of chronic post-traumatic headaches after mild head trauma in US soldiers: an observational study. Headache 2011;51:932-44 CrossRef Medline

8. Bazarian JJ, Zhong J, Blyth B, et al. Diffusion tensor imaging detects clinically important axonal damage after mild traumatic brain injury: a pilot study. J Neurotrauma 2007;24:1447-59 CrossRef Medline

9. Wilde EA, McCauley SR, Hunter JV, et al. Diffusion tensor imaging of acute mild traumatic brain injury in adolescents. Neurology 2008; 70:948-55 CrossRef Medline

10. Alhilali LM, Yaeger K, Collins M, et al. Detection of central white matter injury underlying vestibulopathy after mild traumatic brain injury. Radiology 2014;272:224-32 CrossRef Medline

11. Smith SM, Jenkinson M, Johansen-Berg H, et al. Tract-based spatial statistics: voxelwise analysis of multi-subject diffusion data. Neuroimage 2006;31:1487-505 CrossRef Medline

12. Jolliffe I. Principal component analysis. In: Wiley StatsRef: Statistics Reference Online. New York: John Wiley \& Sons; 2014 CrossRef

13. Headache Classification Subcommittee of the International Headache Society. The International Classification of Headache Disorders: 2nd edition. Cephalalgia 2004:24(suppl 1):9-160 CrossRef Medline

14. Hosmer DW Jr, Lemeshow S, Sturdivant RX. Applied Logistic Regression, 3rd Edition. New York: John Wiley \& Sons; 2013
15. Hulkower MB, Poliak DB, Rosenbaum SB, et al. A decade of DTI in traumatic brain injury: 10 years and $\mathbf{1 0 0}$ articles later. AJNR Am J Neuroradiol 2013;34:2064-74 CrossRef Medline

16. Zhang K, Johnson B, Pennell D, et al. Are functional deficits in concussed individuals consistent with white matter structural alterations: combined FMRI \& DTI study. Exp Brain Res 2010; 204:57-70 CrossRef Medline

17. Kasahara K, Hashimoto K, Abo M, et al. Voxel- and atlas-based analysis of diffusion tensor imaging may reveal focal axonal injuries in mild traumatic brain injury: comparison with diffuse axonal injury. Magn Reson Imaging 2012;30:496-505 CrossRef Medline

18. Messé A, Caplain S, Paradot G, et al. Diffusion tensor imaging and white matter lesions at the subacute stage in mild traumatic brain injury with persistent neurobehavioral impairment. Hum Brain Mapp 2011;32:999-1011 CrossRef Medline

19. Yallampalli R, Wilde EA, Bigler ED, et al. Acute white matter differences in the fornix following mild traumatic brain injury using diffusion tensor imaging. J Neuroimaging 2013;23:224-27 CrossRef Medline

20. Lange RT, Iverson GL, Brubacher JR, et al. Diffusion tensor imaging findings are not strongly associated with postconcussional disorder 2 months following mild traumatic brain injury. J Head Trauma Rehabil 2012;27:188-98 CrossRef Medline

21. Niogi SN, Mukherjee P, Ghajar J, et al. Structural dissociation of attentional control and memory in adults with and without mild traumatic brain injury. Brain 2008;131:3209-21 CrossRef Medline

22. Lipton ML, Gulko E, Zimmerman ME, et al. Diffusion-tensor imaging implicates prefrontal axonal injury in executive function impairment following very mild traumatic brain injury. Radiology 2009;252:816-24 CrossRef Medline

23. Levin HS, Wilde E, Troyanskaya M, et al. Diffusion tensor imaging of mild to moderate blast-related traumatic brain injury and its sequelae. J Neurotrauma 2010;27:683-94 CrossRef Medline

24. Little DM, Kraus MF, Joseph J, et al. Thalamic integrity underlies executive dysfunction in traumatic brain injury. Neurology 2010;74: 558-64 CrossRef Medline

25. Wada T, Asano Y, Shinoda J. Decreased fractional anisotropy evaluated using tract-based spatial statistics and correlated with cognitive dysfunction in patients with mild traumatic brain injury in the chronic stage. AJNR Am J Neuroradiol 2012:33:2117-22 CrossRef Medline

26. Yaeger K, Alhilali L, Fakhran S. Evaluation of tentorial length and angle in sleep-wake disturbances after mild traumatic brain injury. AJR Am J Roentgenol 2014;202:614-18 CrossRef Medline

27. Li XL, Fang YN, Gao QC, et al. A diffusion tensor magnetic resonance imaging study of corpus callosum from adult patients with migraine complicated with depressive/anxious disorder. Headache 2011;51:237-45 CrossRef Medline

28. Yu D, Yuan K, Qin W, et al. Axonal loss of white matter in migraine without aura: a tract-based spatial statistics study. Cephalalgia 2013; 33:34-42 CrossRef Medline

29. Xydis V, Astrakas L, Drougia A, et al. Myelination process in preterm subjects with periventricular leucomalacia assessed by magnetization transfer ratio. Pediatr Radiol 2006;36:934-39 CrossRef Medline

30. Kim SN, Park JS, Jang JH, et al. Increased white matter integrity in the corpus callosum in subjects with high genetic loading for schizophrenia. Prog Neuropsychopharmacol Biol Psychiatry 2012;37: 50-55 CrossRef Medline

31. Schulte T, Maddah M, Müller-Oehring EM, et al. Fiber tract-driven topographical mapping (FTTM) reveals microstructural relevance for interhemispheric visuomotor function in the aging brain. $\mathrm{Neu}$ roimage 2013;77:195-206 CrossRef Medline 